\title{
APPROXIMATE ASSESSMENT OF THE OPERATIONAL PERFORMANCES OF AN UNMANNED AERIAL VEHICHLE ACCORDING TO ITS FLIGHT DATA
}

\author{
Valeriy SILKOV ${ }^{1}$, Mykola DELAS ${ }^{2}$ \\ ${ }^{1}$ Central Scientific-Research Institute of the Armed Forces, Kyiv, Ukraine \\ ${ }^{2}$ National Aviation University, Kyiv, Ukraine \\ E-mail:eduicao@nau.edu.ua
}

Received 18 May 2015; accepted 18 December 2015

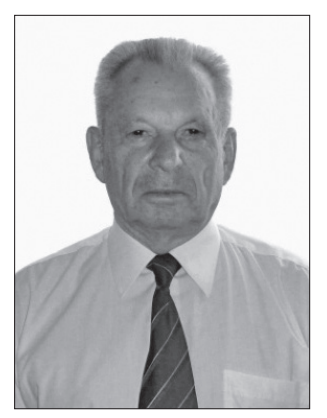

Valeriy SILKOV, Prof.

Education: Kiev Higher Educational Institution of Aviation Military Engineering, Ukraine. Affiliations and functions: researcher at the Central Scientific-Research Institute of the Armed Forces of Ukraine.

Research interests: aerodynamics and dynamics of flight, UAV design.

Publications: author of over 170 scientific papers.

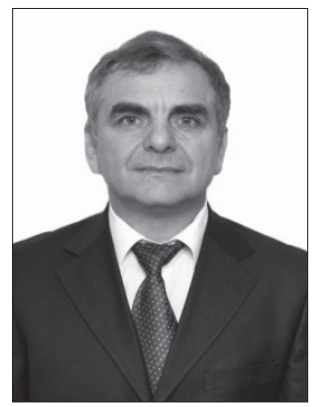

\author{
Mykola DELAS, Assoc. Prof. \\ Education: Kiev Higher Educational Institution of Aviation Military Engineering, Ukraine. \\ Affiliations and functions: associate professor in the Department of Aircraft Control \\ Systems, National Aviation University, Kyiv, Ukraine. \\ Research interests: dynamics of UAV flight. \\ Publications: author of 63 scientific papers.
}

Abstract. The article is dedicated to the substantiation of the complex parameter that characterizes the technical level of an unmanned aerial vehicle (UAV). This parameter includes the maximum lift-to-drag ratio, propeller efficiency, specific fuel consumption, and other components, on which the main flight characteristics, such as flight range and flight duration, depend. To make a comparative assessment of UAVs of different types, a special scale is developed.

Keywords: UAV, lift-to-drag ratio, specific fuel consumption, propeller efficiency, flight range, flight duration, relative stock of fuel, target load, cruising speed, economizing speed.

\section{Introduction}

Unmanned aircraft have become a significant part of the armed forces in all the leading countries of the world in the past 20 years. Their efficiency and practically have been displayed, in all recent military conflicts. Unmanned aerial vehicles, their on-board systems, target load and armament are being continuously improved (Gertler 2012; Goraj 2003; Harrison 2013). Projects to create a standardized interface have been developed in order to increase the promptness of planning to use UAVs in different regions of the world. Therefore, there is a need for the assessment of UAV operational performances (Kharchenko et al. 2013).

Modern unmanned aerial systems are complicated technical devices. They consist of an unmanned aerial vehicle, a power plant, on-board equipment, communication systems, intelligence equipment, and armament. All components require specialized approaches for their effective assessment. To attain this goal, various methods to assess the operational performances of unmanned aerial vehicles and their power plants are taken into consideration. 
2. The calculation of a parameter used for the complex assessment of the efficiency of an unmanned aerial vehicle with a propeller power plant

According to flight dynamics, flight range $L$ and flight duration $T$ are determined by the stock of fuel for horizontal flight $m T$, as well as by fuel consumption, per kilometer $q \mathrm{k}$ and per hour $q \mathrm{~h}$ respectively, as seen in the following equations:

$$
L=\frac{m T}{g k}, \quad T=\frac{m T}{g h},
$$

where $m_{T}=m_{T 0}-m_{T n}$ and $m_{T 0}$ are maximum fueling; $m_{T n}$ is an unproductive fuel consumption (power plant testing, system checking, take-off and climbing, descending and landing, and unproduced fuel remaining).

To take these consumptions into account, it is necessary to establish a special coefficient, for instance $m_{T}=0,94 m_{T 0}$. Fuel consumption per hour, in turn, depends on specific consumption $C_{e}$ (fuel consumption for 1 horse power per 1 hour of power plant operation), as well as on the effective power on the shaft of engine $N_{e}$ :

$$
q_{h}=C_{e} N_{e} .
$$

The main factors that influence the value of $C_{e}$ are the rotational speed of a shaft (revolutions per minute), height $H$ and flight speed $V$. Dependence $N_{e}(n)$ and dependence $C_{e}(n)$ are called 'throttle characteristics'. They are shown in their typical form in Figure 1. According to this figure, the engine power increases simultaneously with the acceleration of revolutions from the minimum to a standard rate. Further acceleration of revolutions can lead to an opposite effect.

For some engines, the rated speed can be equal to the maximum one. Thus, the nominal mode is usually the most economizing at the corresponding speed and altitude. It should be noted that the maximum range flight mode is similar to the nominal mode.

With increasing altitude, air density $\rho$ decreases and the amount of fuel mixture supplied to the engine cylinder decreases, consequently the engine power is reduced. Variations in the maximum power depending on height $H$ up to 11000 meters and engine speed $n$ can be estimated using the following formula:

$$
N_{H}=N_{0}\left(1-\frac{H}{44308}\right)^{4.255}, N=k_{N} n^{3},
$$

where $N_{0}$ is the maximum power on the ground, $H$ is altitude in meters.

The coefficient of proportionality between power and speed can be determined by the parameters of the maximum mode at an appropriate height. The rate of engine throttling is usually defined taking into consideration either power or speed.

Specific fuel consumption changes depending on height and power. Height influences specific fuel

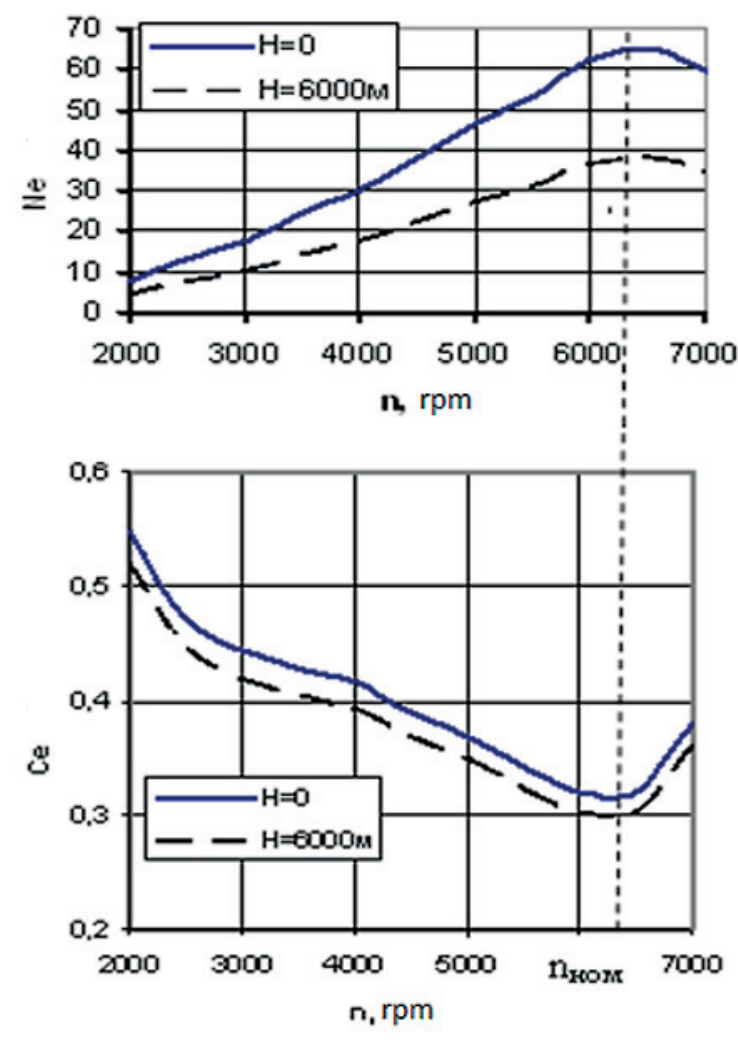

Fig. 1. Throttle characteristics of an engine

consumption depending on the air temperature, i.e. with height increasing up to 11,000 meters, fuel consumption decreases, and then remains constant, under the condition that $T_{H>11 к м}=$ const. As seen from Figure 1 , an increase in height from 0 to 6000 meters results in only a $15 \%$ decrease of the value of $C_{e}$.

Considering UAVs which have a range of altitude equal to 4000 meters at maximum, the influence of height on specific fuel consumption can be neglected and specific fuel consumption, in turn, can be considered to be independent of height. Regarding high-altitude UAVs, the influence of height ought to be taken into account. When operating an aircraft in a straight and horizontal flight, it is affected by drag force $X_{a}$, which can be expressed in Newtons or kilograms through gravity $g m$ (equal to the lifting force) and lift-to-drag ratio $K$ :

$$
X_{a}=\frac{g m}{K}[\mathrm{~N}]=\frac{m}{K}[\mathrm{~kg}] .
$$

Multiplying this force by the flight speed, the result equals the required power $N_{\Pi}$, which is usually expressed in kilowatts or horsepower:

$$
N_{\Pi}=10^{-3} \frac{g m V[\mathcal{M} / c]}{K}[\mathrm{~kW}]=\frac{m V[\kappa M / \text { uac }]}{270 K}[\text { r.c. }]
$$

Flight speed can be measured in meters per second or in kilometers per hour. Equation (1.5) shows an obvious connection between a kilowatt and horsepower, which is as follows: $1 \mathrm{~kW}=0.735 \mathrm{hp}$. In some references, kilowatts are used instead of horsepower. 


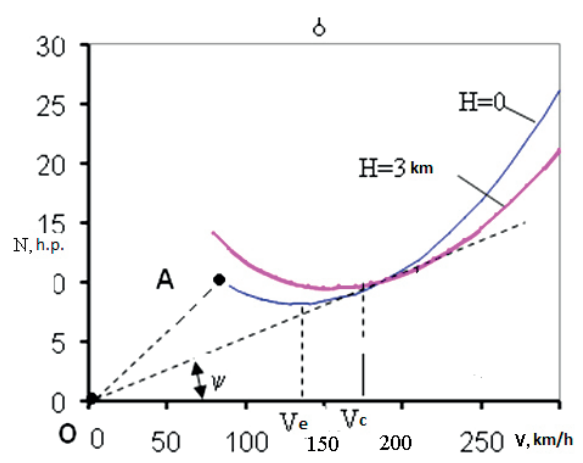

Fig. 2. Dependence of required power on flight speed at different altitudes

Figure 2 shows the dependence of the required power on the flight speed for altitudes from 0 to 3000 meters. The power strongly depends on flight speed: $N_{\Pi}=X_{a} V=C_{x a} \rho V^{2} S V / 2=C_{x a} \rho V^{3} S / 2$. Its minimum value corresponds to the cruising speed (cruising angle of attack and cruising coefficient of lifting force $C_{y e}$ ).

The dependence of required power on speed $N_{\Pi} / V$ at any point of the diagram in Figure 2 (e.g. at point A) is equal to drag force $\mathrm{X} a$. In the diagram, this dependence is expressed as the tangent $\psi$ of the inclination angle of beam $O A$ to the axis of speeds.

Obviously, this angle is minimal in case line $O A$ that touches the curve of required power. The point of tangency corresponds to the cruising speed $V_{R S}$, as well as cruising angle of attack $\alpha_{R S}$, cruising coefficient of lifting force $C_{y R S}$ and maximum lift-to-drag ratio $K_{\max }$. These characteristics are determined by the polar curve, which usually has a quadratic dependence. Then the cruising coefficient of lifting force is expressed with parameters of the polar curve:

$$
C_{x a}=C_{x 0}+A C_{y a}^{2}, \quad C_{y R S}=\sqrt{\frac{C_{x 0}}{A}} .
$$

According to Iliushko, Silkov (2010), $C_{y R S}$ is an essential characteristic of optimal flight modes. Using the required power and the coefficient of propeller efficiency $\eta_{B}$, formula (1.2) can be rewritten as follows:

$$
q_{h}=C_{e} \frac{N_{\Pi}}{\eta_{B}}=\frac{C_{e} m V[\kappa M / u]}{270 \eta_{B} K}[\kappa 2 / u] .
$$

Upon contemplating this formula, it is clear that the flight mode and, especially, the speed and angle of attack can significantly affect value $q_{h}$. However, the determination of the minimum hourly consumption is of the greatest interest.

Formula (1.7) also shows that the minimum hourly fuel consumption should correspond to the minimum ratio $V / K$. According to this condition, the flight mode can be determined. To do this, the expression for the speed of a straight horizontal flight is taken and divided by quality $K=C_{y a} / C_{x a}$ :

$$
\frac{V}{K}=\sqrt{\frac{2 g m}{C_{y a} \rho S}} \frac{C_{x a}}{C_{y a}}=\sqrt{\frac{2 g m}{\rho S}} \frac{C_{x a}}{C_{y a}^{3 / 2}} .
$$

Obviously, the ratio $V / K$ is minimal if value $\frac{C_{x a}}{C_{y a}^{3 / 2}}$ is minimal at the cruising angle of attack (or cruising value $C_{\text {yek }}$ ). The flight mode can be determined correspondingly to the condition given above. To do this, polar curve equation (1.6) is divided by value $C_{y a}^{3 / 2}$ :

$$
\frac{C_{x a}}{C_{y a}^{3 / 2}}=\frac{C_{x 0}}{C_{y a}^{3 / 2}}+\frac{A C_{y a}^{2}}{C_{y a}^{3 / 2}} .
$$

After differentiating the right side of equation (1.9) by value $C_{y a}$ and equating it to zero, the expression for the cruising coefficient of lifting force is obtained as follows:

$$
C_{y e \kappa}=\sqrt{3 \frac{C_{x 0}}{A}}=\sqrt{3} C_{y н в}=1,73 C_{y н в} .
$$

Value $C_{\text {yeк }}$ turned out to exceed the cruising value 1.73 times. If the speed of horizontal flight is inversely proportional to the square root of the coefficient of lifting force, the ratio between cruising speed and economizing speed is described as follows:

$$
V_{H B}=\sqrt[4]{3} V_{e \kappa}=1.32 V_{e \kappa} .
$$

The coefficient of drag at the cruising angle of attack is calculated according to the following equation:

$$
C_{x e \kappa}=C_{x 0}+A C_{y e \kappa}^{2} \text {. }
$$

Taking into account equation (1.10) and the fact that $C_{x 0}=A C_{R S}^{2}, K_{R S}=C_{y R S} /\left(2 C_{x 0}\right)$, the lift-to-drag ratio in the economizing mode is calculated as follows:

$$
K_{e \kappa}=\frac{\sqrt{3}}{2} K_{\max }=0.866 K_{\max } .
$$

Thus, the connection between the economizing flight mode and cruising flight mode is determined by equations (1.10), (1.11), (1.12), and (1.13). In the cruising mode, the lift-to-drag ratio is $13 \%$ greater than in the economizing mode. With increasing altitude, the curves of required power rise up and move to the right; consequently, the economizing speed increases at a constant attack angle (lift-to-drag ratio).

Engine power reduction due to air density with increasing altitude has to be compensated with an increase in the engine speed (revolution per minute); consequently, value $C_{e}$ decreases. At a certain height, the engine is stabilized to its nominal speed (revolution per minute). This flight mode corresponds to the lowest hourly fuel consumption when value $C_{e}$ reaches the minimum. Lift-to-drag ratio is described as $K_{e \kappa}$, and the propeller efficiency should also be maximized under the terms of its selection. In this case, the maximum flight duration may be achieved. Hourly consumption and fuel supply are the parameters that make it possible to determine the maximum flight duration:

$$
\begin{aligned}
& T_{\max }=\frac{m_{T}}{q_{h \min }}=\frac{270 \eta_{b} K_{e \kappa} m_{T}}{C_{e \min } m V_{e \kappa}}= \\
& \frac{270 \eta_{\varepsilon}\left(0,866 K_{\max }\right) m_{T}}{C_{e \min } m V_{e \kappa}}=\frac{234 \eta_{b} K_{\max } m_{T}}{C_{e \text { mim }} m V_{e \kappa}} .
\end{aligned}
$$


In equation (1.14), value $K_{e \kappa}$ is replaced by value $K_{\max }$ in accordance with equation (1.13). On the basis of these two equations, the following equation is formulated:

$$
k_{e}=\frac{K_{\max } \eta_{\text {e max }}}{C_{e \min }},
$$

Value $k_{e}$ can be presented as the efficiency factor of a UAV. The reason for this is that the efficiency factor is applied for a complex estimation of a UAV, in particular a glider (via its lift-to-drag ratio), the propeller (via its efficiency) and power plant (via the specific fuel consumption). The dimension of value $k_{e}$ is inverse to the dimension $C_{e}$. In most cases, it is expressed in horsepower per hour in respect to a kilogram of fuel. Taking into consideration that all the components of equation (1.15) are relative values, they are supposed to be convenient for practical use. Using equations (1.14) and (1.15), the maximum flight duration can be determined as follows:

$$
T_{\max }=\frac{234 k_{e} m_{T}}{m V_{e \kappa}}=\frac{234 k_{e} \bar{m}_{T}}{V_{e \kappa}[\kappa M / u]}
$$

where $\bar{m}_{T}=\frac{m_{T}}{m}$ is the relative amount of fuel for horizontal flight, and $m$ is the average weight of the aircraft in flight.

Thus, the maximum flight duration is determined by two parameters presented: the efficiency factor and the relative amount of fuel. Flight speed affecting the required power is characterized by the kinematic parameters. As shown above, to achieve the maximum flight duration, flight speed should be efficient, and the engine speed, in turn, should be close to the nominal one. Equation (1.16) makes it possible to obtain an expression for the maximum value of the efficiency factor which is determined depending on the flight duration. This expression is described as follows:

$$
k_{e}=\frac{T_{\max } V_{e \kappa}}{234 \bar{m}_{T}}
$$

Having been obtained equation (1.17) makes it possible to calculate the maximum value of coefficient $k_{e}$ using cruising speed $V_{c r}$, maximum flight duration $T_{\text {max }}$, and the relative weight $\bar{m}_{T}$ of fuel. In contrast with expression (1.15), all the parameters of expression (1.17), as a rule, are provided by the manufacturers of UAVs. Moreover, they are supported by the flight experiment and have a higher degree of confidence than blowing and design parameters. Thus, coefficient $k_{e}$ is determined by equation (1.17), i.e. by the averaged flight parameters. This coefficient objectively characterizes the operational performance of a UAV, as it takes into account the error of piloting, deviations from the optimal trajectory for various reasons, and so on. Fuel consumption per kilometer is connected with the hourly consumption, as seen in the following ratio: $q_{k}=q_{h} / V$. Fuel consumption per kilometer and fuel consumption at economizing speed are calculated as follows:

$$
q_{h R S}=\frac{C_{e} m V_{R S}}{270 \eta_{B} K_{\max }}, \quad q_{\kappa}=\frac{C_{e} m}{270 \eta_{B} K_{\max }} .
$$

Obviously, $q_{\kappa}$ does not depend on the speed, but inexplicitly affects fuel consumption per kilometer via lift-to-drag ratio. Flight at cruising speed provides the maximum quality and minimum fuel consumption per kilometer:

$$
L_{\max }=\frac{m_{T}}{q_{k \min }}=270 \bar{m}_{T} \frac{K_{\max } \eta_{B}}{C_{e}}=270 \bar{m}_{T} k_{e}
$$

Analyzing expression (1.19), it is obvious that the efficiency factor is calculated taking into account the flight range:

$$
k_{e}=\frac{L_{\max }}{270 \bar{m}_{T}} \text {. }
$$

Expression (1.20) allows determining the efficiency factor according to the maximum flight range and the relative stock of fuel, i.e. it is equal to the maximum flight range divided into 270 relative stocks of fuel.

Upon comparing expressions (1.17) and (1.20), it is found that $T_{\text {max }} V_{e \kappa}=0.866 \mathrm{~L}_{\text {max }}$. The given equality defines a difference in flight ranges at efficient and cruising speeds, i.e. flight at cruising speed allows obtaining the maximum flight range which is approximately $13 \%$ greater than the maximum one at economizing speed, however the flight duration is smaller.

It is necessary to emphasize that the values calculated from expressions (1.15), (1.17) and (1.20) should lead to an identical result, and the possibility of their calculation depends on the availability of particular initial data. Thus, coefficient $k_{e}$ can be used as an absolute parameter of UAV operational performances, and also as an integrated quality parameter when assessing UAV samples in accordance with the technique given in Mitrakhovich, Silkov (2012).

\section{Rating scales to assess the parameter of operational performance of UAVs with propeller power plants.}

To check the operating capacity of the proposed parameters, the possibility of their application is estimated to determine the flight qualities of available and perspective UAVs. Some references to this paper, namely Iliushko, Silkov (2010) and Daly (2011), and the Internet were used as the main sources of information concerning UAV flight characteristics. A group of UAVs on which the full data are obtained has been selected. Initial characteristics of the selected UAVs are shown in Table 1. 
Table 1. Results obtained upon calculating coefficient $k_{e}$ for UAVs with piston engines

\begin{tabular}{|c|c|c|c|c|c|c|c|c|c|c|c|c|}
\hline \multirow{2}{*}{ UAV name } & $\mathrm{L}_{\max }$ & $\mathrm{T}_{\max }$ & $V_{\text {крс }}$ & $\mathrm{H}_{\mathrm{cT}}$ & $\mathrm{N}_{0}$ & $\mathrm{~m}_{\text {взл, }}$ & $\mathrm{m}_{\mathrm{T}}$ & $\mathrm{m}_{\mathrm{T}} / \mathrm{m}$ & $\mathrm{q}_{\mathrm{h}}$ & ke & $\mathrm{C}_{\mathrm{eH}}$ & $\mathrm{K}_{\max }$ \\
\hline & $\mathrm{km}$ & $\mathrm{h}$ & $\mathrm{km} / \mathrm{h}$ & $\mathrm{m}$ & h.p. & $\mathrm{kg}$ & $\mathrm{kg}$ & & $\mathrm{kg} / \mathrm{h}$ & h.p., h/kg & kg/h.p.,h & \\
\hline Scout & & 7 & 102 & 4575 & 22 & 159 & 25 & 0,17 & 3,6 & 14 & 0,41 & 6,9 \\
\hline Ranger & 510 & 3 & 180 & 4500 & 38 & 275 & 40 & 0,16 & 13,3 & 11 & 0,87 & 12,2 \\
\hline Eagle Eye & & 6 & 370 & 6100 & 641 & 1020 & 378 & 0,45 & 63,0 & 16 & 0,29 & 5,7 \\
\hline Predator & & 24 & 130 & 9620 & 115 & 1020 & 300 & 0,34 & 12,5 & 29 & 0,48 & 17,7 \\
\hline Pioneer & & 5 & 120 & 4570 & 26 & 205 & 35 & 0,19 & 7,0 & 10 & 0,68 & 8,8 \\
\hline Searcher II & & 14 & 196 & 5000 & 47 & 436 & 110 & 0,29 & 7,9 & 31 & 0,44 & 16,9 \\
\hline Hunter 5B & 1897 & 18 & 165 & 5490 & 56 & 817 & 127 & 0,17 & 7,1 & 57 & 0,35 & 24,9 \\
\hline Shadow 7B & & 7 & 110 & 4570 & 38 & 170 & 33 & 0,21 & 4,7 & 12 & 0,31 & 4,5 \\
\hline Reaper RQ9 & 5920 & 14 & 160 & 15000 & 910 & 4763 & 1300 & 0,32 & 92,9 & 23 & 0,92 & 26,3 \\
\hline Long Gun & & 30 & 230 & 4546 & 28 & 328 & 136 & 0,52 & 4,5 & 43 & 0,41 & 21,7 \\
\hline Neptune & & 5,5 & 112 & 2425 & 15 & 176 & 8 & 0,05 & 1,5 & 43 & 0,2 & 10,5 \\
\hline Strepet-S & 1300 & 12 & 150 & 4500 & 28 & 180 & 52 & 0,34 & 4,3 & 17 & 0,39 & 8,3 \\
\hline Hermes-450 & & 20 & 130 & 6000 & 52 & 450 & 105 & 0,26 & 5,3 & 32 & 0,3 & 11,8 \\
\hline Mirach-26 & & 6 & 170 & 4000 & 27 & 230 & 24 & 0,11 & 4,0 & 30 & 0,35 & 13,1 \\
\hline Strepet-L & & 6 & 140 & 4500 & 15 & 95 & 12 & 0,13 & 2,0 & 20 & 0,33 & 8,4 \\
\hline Remez-3 & & 2 & 80 & 2000 & 2,48 & 10 & 1,5 & 0,16 & 0,8 & 3 & 0,58 & 2,3 \\
\hline Dozor-100 & 1200 & 10 & 130 & 4500 & 19 & 95 & 24 & 0,29 & 2,4 & 15 & 0,31 & 5,7 \\
\hline Dozor-600 & 3700 & 24 & 140 & 7500 & 115 & 640 & 160 & 0,29 & 6,7 & 38 & 0,2 & 9,6 \\
\hline
\end{tabular}

Taking into consideration that the proposed parameters are applied to assess UAV operational performance, their types and dimensions do not particularly have any significance. In this case, only UAVs with propeller engine power plants and UAVs with 2 and 4-cycle petrol engines are selected.

Having analyzed the components of coefficient $k_{e}$, as seen in equation (1.15), it is evident that all of them vary in rather narrow limits. So, the maximum rating of propeller efficiency theoretically equals 1 , and practically does not exceed 0,9 . The maximum lift-to-drag ratio of traditional UAV schemes is usually not more than 20 , but for the majority of existing UAVs - not more than 15. The minimum specific fuel consumptions of petrol engines lay within the limits of $0,2 \ldots 0,5 \mathrm{~kg} / \mathrm{h} . \mathrm{p}$.,h. Therefore, theoretically the maximum value $k_{e}$ should not exceed 80 h.p., h/kg, and practically should not be more than 50 h.p., h/kg.

To carry out the comparative assessment, it is necessary to select UAV samples close to the best ones with the following characteristics: $K_{\max }=15, \eta_{B}=0,9$, $C_{e \text { min }}=0,27 \mathrm{~kg} / \mathrm{h} . \mathrm{p}$., $\mathrm{h}$, and to consider them as the standard. Under these conditions, value $k_{e}$ is equal to 50 h.p., h/kg. This value is considered to be the basic for an estimation of existing UAVs and can be used for their comparison.

Table 1 shows the results of calculation of coefficient $k_{e}$ for each selected group of UAVs, according to the published data of their parameters. The calculations were conducted for two versions of flights: maximum duration flights and maximum range flights. As the initial information on range flights is extremely limited, Table 1 includes only the results pertaining to the flight duration. When conducting these calculations, UAVs of various types were selected, commencing with "Remez-3", a small vehicle weighed at $10 \mathrm{~kg}$, to strategic UAVs such as "Eagle Eye". Due to to value $k_{e}$, it is possible to determine the technical level of the selected vehicles.

To perform more traditional estimations, it is necessary to construct a rating scale (for instance, a fivepoint range), by dividing a 50-point scale into groups. The example of such a scale is shown in Table 2. Use of the given scale makes it possible to rank the vehicles given in Table 1.

After calculating coefficient $k_{e}$ for vehicles of a specific group, it is possible to determine the best UAV sample or to analyze the reasons of the low efficiency of some vehicles. So, Table 1 demonstrates that super-efficient UAVs may only include Hunter 5B. Vehicles with 5 points include Long Gun and Neptune, vehicles with 4 points - Picket-600 and Hermes-450, and vehicles with 1 point - Remez-3.

It is recommended to critically view the given estimations. Their value depends exclusively on the input data which can differ from real data due to the low accuracy of flight parameters (for example, flight range or flight duration), as well as due to intentionally overrated data for a certain purpose. That is why, in case of doubt, the obtained results ought to be checked again. 
Table 2. The normalized rating scale of UAV operational performance

\begin{tabular}{|c|l|l|}
\hline Number of points & \multicolumn{1}{|c|}{ Value $\mathrm{k}_{\mathrm{e}}, \mathrm{h} . \mathrm{p} ., \mathrm{h} / \mathrm{kg}$} & \multicolumn{1}{c|}{ Description } \\
\hline 1 & 10 and less & $\begin{array}{l}\text { Insufficient aerodynamic configuration, inefficient engine, the propeller is not } \\
\text { selected well }\end{array}$ \\
\hline 2 & $11 \ldots 20$ & $\begin{array}{l}\text { The engine and propeller do not comply with the characteristics of a glider, } \\
\text { significant fuel consumption }\end{array}$ \\
\hline 3 & $21 \ldots 30$ & $\begin{array}{l}\text { Significant lift-to-drag ratio, insignificant fuel consumption, acceptable } \\
\text { propeller efficiency }\end{array}$ \\
\hline 4 & $31 \ldots 40$ & $\begin{array}{l}\text { Significant flight range and flight duration, new technologies are implemented } \\
\text { Modern materials, manufacturing technologies, and achievements in the } \\
\text { field of aerodynamics, engine design and aircraft propeller manufacturing are } \\
\text { implemented }\end{array}$ \\
\hline 5 & $41 \ldots 50$ &
\end{tabular}

Note: UAVs with $k_{e}>50$ h.p.,h/kg may be included into the group of super-efficient vehicles.

Table 1 can also help to reveal erroneous data or to analyze the "bottlenecks" in a UAV layout and in a power plant. For instance, it was revealed that UAV "Ranger" had excessively high specific fuel consumption. This fact proves that the demands for a stock of fuel are mostly overrated. For the same reason, it is necessary to consider specific fuel consumption for the Dozor-600 to be underestimated as well.

The proposed parameters of UAV efficiency can be widely used in practice in order to carry out a fast estimation without the application of complicated programs. If necessary, it is possible to operatively construct the recommended tables for a group of vehicles for their estimation when purchasing foreign samples, selecting the manufacturer, and also for the solution of specific tactical tasks, etc. The parameters given above can be used to develop some standards and tasks in the area of UAV operation.

\section{Estimation of the reliability of input data obtained on UAVs with propeller power plants}

While determining the efficiency factor by equations (1.17) and (1.20), the precision depends on the accuracy of the main parameters, such as flight duration, flight range, and fuel consumption for flight performance. As it has been told earlier, this data can be casually or intentionally distorted. To check its reliability, it is reasonable to use other sources of information, in particular the socalled indirect methods of estimation. For instance, to redefine value $k_{e}$, it is recommended to use some power plant data, its power and specific fuel consumption to be exact.

The obligatory list of initial UAV characteristics, as a rule, includes the starting capacity of power plant $N_{0}$. The maximum power of isolated engine Nen may be specified as well. These powers are connected with each other by the ratio $N_{0}=N_{e n} . \eta_{p} \kappa_{p}$, where $\eta_{p}$ is the propeller efficiency and $\kappa_{p}$ is the coefficient considering the power loss of an engine while being installed on a UAV. As usual $\kappa_{p}=0,9 \ldots 0,95$.
Having analyzed equation (1.15), it is possible to draw a conclusion that the key source of errors when determining coefficient $\kappa_{e}$ is the lift-to-drag ratio. To implement its maximum value in flight, it is necessary to ensure, first, the cruising attack angle by means of providing a specified altitude and speed. Thus, the significant deflection of revolutions from the specified ones under the control of variable flight speed leads to an extreme fuel consumption (increasing value $C_{e}$ ). It is obvious that in conditions of even a small turbulence, it is uneasy for the operator to perform a UAV flight in a specified mode.

Consequently, the "flight" value of the maximum lift-to-drag ratio can appear to be significantly smaller than its real value, as it depends not only on the specified parameters, but also on the manner of piloting the vehicle. At the same time, the mean value of the lift-todrag ratio has an advantage, because it pertains to values that can be implemented in practice.

The maximum lift-to-drag ratio can be obtained from the equation (1.14) as follows:

$$
K_{\max }=\frac{T_{\max } V_{e k} C_{e \min }}{234 \eta_{\hat{a}} \bar{m}_{T}} .
$$

Equation (1.21) makes it clear that if the task on flight duration and the stock of fuel is specified accurately, the key source of errors influencing the value of the lift-to-drag ratio is specific fuel consumption and propeller efficiency. The specific fuel consumption can be obtained:

- by the throttle characteristics;

- by the mean fuel consumption per hour.

In the first case, according to a specified throttle characteristic, it is necessary:

- to revise the minimum value $\bar{C}_{e \text { min }}$;

- to refer to its absolute value for zero altitude $C_{e 0 \min }=\bar{C}_{e \min } C_{e 0 \max }$, where value $C_{e 0 \max }$ corresponds to the mode of the maximum engine power on the ground; 
- to calculate the minimum specific fuel consumption at a specified altitude.

$$
C_{e \min }=C_{e 0 \min }\left[1-\frac{H}{44308}\right]^{0.5} \text {. }
$$

To determine the specific fuel consumption by fuel consumption per hour, it is necessary:

- to determine the mean fuel consumption per hour for a flight $q_{h}=m_{T} / T_{\text {max }}$;

- to determine maximum engine power $N_{H}$ at a specified altitude according to equation (1.3);

- to determine the throttle ratio of the engine according to revolutions, meaning that nominal revolutions $n_{\text {nom }}=(0,8 \ldots 0,9) n_{\text {max }}$;

- to determine available engine power $N_{e}$, meaning that power $N_{e}=(0.5 \ldots 0.7) N_{H}$ corresponds to the accepted revolutions;

- to calculate real specific fuel consumption $C_{e}=$ $q_{h} / N_{e}$.

The propeller is usually selected taking into account the capacity of the power plant and the revolutions, so that its efficiency is the maximum in the main flight modes. For existing UAV propellers, value $\eta_{b}=0,7 \ldots 0$, 8 and less. This value can be determined according to the certain type and geometry of a propeller.

After defining values $C_{e}$ and $\eta_{b}$, equation (1.21) allows calculating the maximum lift-to-drag ratio. Its value as a first approximation allows to contemplate the issues concerning the reliability of the obtained calculations of coefficient $\kappa_{e}$, and to reveal possible errors in calculations.

According to the statistics, the lift-to-drag ratio of traditional UAV schemes does not exceed 15 (20). That is why the significant excess of the given values indicates a gross error in input data or methods of calculation and requires searching for more reliable sources of information. It is obvious that while determining the lift-to-drag ratio, the significant error occurs due to the capacity of a power plant. However, if the capacity is adequately and accurately calculated, the lift-to-drag ratio is barely approximate (Austin 2010). Consequently, it can be concluded that there is precision in the calculations of the lift-to-drag ratio obtained during the flight experiment, so this data can be considered reliable.

Using equation (1.15), it is possible to clarify value $k_{e}$, and then to recalculate the flight range and flight duration. Several iterations help to achieve a significant increase in the accuracy of the final results. According to the proposed method, the calculation of specific fuel consumption and the maximum lift-to-drag ratio is carried out. The results of the calculation are given in Table 1. Analysing this data, a number of inconsistencies can be revealed. For example, both parameters appeared to be strongly overestimated for UAV "Reaper RQ-9".
Apparently, this overestimation is connected with the mixture of various modifications and inconsistent data obtained from different sources of information.

\section{Conclusions}

1. The paper has substantiated the complex parameter of UAV operational performances, as well as the efficiency factor which integrates the most important UAV qualities, such as aerodynamics and airframe configurations (determined by the maximum liftto-drag ratio), thrust capabilities of a propeller (determined by its efficiency) and efficiency of a power plant (determined by the specific fuel consumption).

2. The efficiency factor combined with a relative stock of fuel determines the main flight characteristics of a UAV, such as flight range and flight duration.

3. The efficiency factor as a separate characteristic of UAV operational performance can be determined at the design stage by the maximum lift-to-drag ratio, propeller efficiency and specific fuel consumption or at the stage of flight tests by the maximum flight duration and flight range.

4. The efficiency factor can be used for a comparative estimation of operational performance of each UAV from a particular group and the selection of the best samples.

\section{References}

Austin, R. 2010. Unmanned aircraft systems. UAVs design, development and deployment. $1^{\text {st }}$ ed. Chichester: John Wiley and Sons Ltd. 288 p. http://dx.doi.org/10.1002/9780470664797

Daly, M. 2011. Unmanned aerial vehicles and targets. Coulsdon: Jane's Information Group. 348 p.

Gertler, J. 2012. U.S. Unmanned aerial systems. Congressional Research Service 7-5700 Report for Congress R42136.

Goraj, Z. 2003. Civilian and military unmanned aerial vehicles - overview of European and American effort and challenges for the future, Aviation 7(1): 3-15.

Harrison, G. J. 2013. Unmanned Aircraft Systems (UAS): manufacturing trends. Congressional Research Service 7-5700 Report for Congress R42938.

Iliushko, V.; Silkov, V. 2010. Unmanned Aerial Vehicles: methods of approximate calculations of the main parameters and characteristics. Kiev: the Press of the Central ScientificResearch Institute of the Armed Forces of Ukraine. 302 p.

Kharchenko, O.; Bohaslavets, S.; Kotsurenko, Y. 2013. Comprehensive analysis of the prospects of development of military unmanned aircraft in the armed forces of the leading world countries, Science and Defense 1: 23-27.

Mitrakhovich, M.; Silkov, V. 2012. Unmanned aircraft systems: methods of comparative assessment of combat capabilities. Kiev: The Press of the Central Scientific-Research Institute of the Armed Forces of Ukraine. 302 p. 